\title{
Outcomes for women admitted to a mother and baby unit: a systematic review
}

\author{
This article was published in the following Dove Press journal: \\ International Journal of Women's Health \\ 30 April 2015 \\ Number of times this article has been viewed
}

\section{Rebecca Gillham' \\ Anja Wittkowski ${ }^{1,2}$}

'School of Psychological Sciences, University of Manchester, ${ }^{2}$ Manchester Mental Health and Social Care Trust,

Manchester, UK
Correspondence: Anja Wittkowski School of Psychological Sciences, University of Manchester, 2nd floor, Zochonis Building, Brunswick Street, Manchester MI 3 9PL, UK

Tel +44 I6I 3060400

Fax +44 I6। 306040 I

Email anja.wittkowski@manchester.ac.uk
Abstract: Mother and baby units (MBUs) provide inpatient psychiatric care for mothers and their infants up to a year after childbirth. They are commissioned to support the mother-infant relationship as well as stabilize maternal mental health. As their efficacy at meeting these aims had not previously been systematically assessed, this paper reviewed the international literature relating to psychological outcomes following MBU admission. A systematic search of five databases identified 23 papers eligible for inclusion, reporting on a range of outcomes indicating positive effects on maternal mental health and the mother-infant relationship and an absence of adverse effects on child development. The review also highlighted specific groups responding less favorably to MBU admission, eg, mothers with a diagnosis of schizophrenia. Although the included studies were of variable methodological quality, the research findings consistently indicated positive effects. Implications for research and clinical practice are outlined in the discussion.

Keywords: mother and baby unit, systematic review, psychological outcomes, postnatal mental health, quality assessment, mothers

\section{Introduction}

Recent figures indicate approximately $20 \%$ of women experience postpartum mental health difficulties, ${ }^{1}$ and four in every 1,000 women require inpatient admission postnatally. ${ }^{2}$ Specialist mother and baby units (MBUs) provide joint admissions of mothers and babies for inpatient treatment and monitoring of the mother-infant relationship. MBU provision varies internationally. Currently, there are MBUs in the UK $(n=17)$, France, Belgium, Germany, and Australia. ${ }^{1}$ The US and India have MBUs, but they are fewer in number. ${ }^{3}$ Some units are mother-baby facilities (MBFs), which are general psychiatric units with capacity for joint admissions, not dedicated MBUs. "MBU" is used to refer to both MBUs and MBFs throughout this review.

Main ${ }^{4}$ observed that separating the mother and infant during the 1st year could impact negatively on the developing attachment relationship. Joint admission, hypothesized to be beneficial for both parties, allows for the observation of the mother caring for her infant and for a thorough risk assessment. Consequently, MBUs have been recommended in clinical guidelines internationally. The National Institute for Health and Care Excellence ${ }^{5}$ outlined the role of MBUs in managing mental health problems during pregnancy, assessing mental illness, risks, and parenting skills, and providing expert care for the mother and infant. Similarly, the Scottish Intercollegiate Guidelines Network $^{6}$ recommended mothers have the option of joint MBU admission. However, over the 50 years since Main's ${ }^{4}$ initial observations, little has been published regarding the effect of MBU treatment. To date, no reviews have evaluated its psychological outcomes, despite the important clinical treatment these units provide for mothers in crisis. 


\section{Aims}

The aim of this systematic review was to evaluate the psychological outcomes of MBU admission: 1) by addressing how admission impacted on maternal mental health, motherinfant relationships, and child health and development, and 2) by evaluating the methodological robustness of the research. Further aims included identifying the commonly used outcome measures and exploring the efficacy of particular MBU treatments.

\section{Literature search Strategy and findings}

A systematic search used Ovid to review five databases from inception to January 2, 2015: PsycInfo, Medline, Embase, Health Management Information Consortium, and Maternity and Infant Care. Furthermore, reference lists of included papers were hand-searched for relevant articles, and citation searches were completed on included papers.

Search terms included "mother and baby unit\$" OR "mother-baby unit\$" OR "postnatal mental health\$” OR “mother-baby psychiat\$” OR “mother-infant unit\$” OR "postpartum depressi\$” OR "postpartum psychos\$” OR "perinatal psychia\$” OR "postnatal psychia\$” OR "postpartum psychia\$” AND "outcome\$” OR "maternal clinical outcome\$” OR “parenting outcome\$” OR “attachment\$” OR "bond\$” OR “mother-infant interaction\$”. Figure 1, based on Preferred Reporting Items for Systematic Reviews and Meta-Analyses ${ }^{7}$ guidelines, outlines the search process.

The Quality Assessment Tool for Studies with Diverse Designs (QATSDD) was chosen because the methodologies used in these studies were expected to be diverse. ${ }^{8}$ Each of the 14 QATSDD items related to quantitative studies was rated on a 4-point scale from "not at all" (0) to "complete" (3). Consistent with other uses of this measure, percentage scores were reported, calculated using the actual score and the maximum total score of 42 . Papers scoring over $75 \%$ were considered "high" quality, those between $50 \%$ and $75 \%$ "good", 25\%-50\% "moderate", and below 25\% "poor". The first author and a peer, independent to the study team, independently rated all papers and resolved any discrepancies through discussion. Interrater reliability was very $\operatorname{good}(\kappa=0.91)$. The relevant information is summarized in Tables 1 and 2.

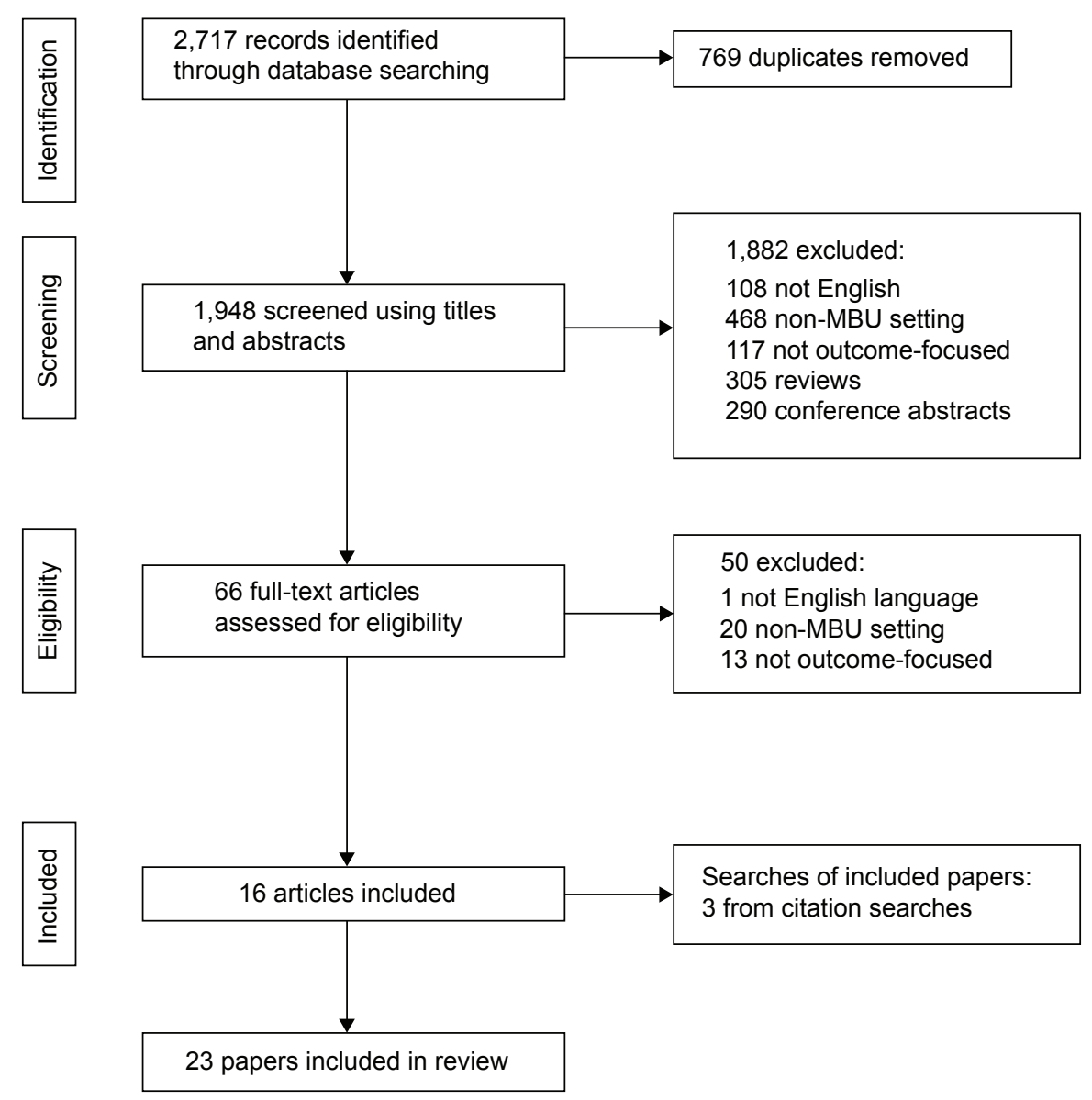

Figure I Flowchart demonstrating literature-review procedure. Abbreviation: MBU, mother and baby unit. 


\section{Inclusion and exclusion criteria}

Inclusion criteria were: 1) English language, 2) published in a peer-reviewed journal, 3) reporting outcomes relating to women admitted to a psychiatric MBU, 4) assessing maternal well-being, the mother-infant relationship, child development, or another psychological outcome, and 5) including assessment of change over time or functioning at discharge. Reviews were excluded. Studies on child-care arrangements ${ }^{9}$ and relapse rates ${ }^{10}$ were also excluded, as were studies in "mothercraft" units. ${ }^{11}$

\section{Search results Selection of studies}

A total of 23 papers were identified for inclusion in this review.

\section{$\mathrm{MBU} / \mathrm{MBF}$ characteristics}

The units ranged from four to 13 beds, and had multidisciplinary staff teams (Table 1). As information about treatments offered was sparse, collating comparable findings was difficult. Only eight papers described psychological treatments that focused on the mother or the mother-infant relationship.

\section{Characteristics of participants}

This review is based on 5,023 participants, including 215 control participants $(4.3 \%)$. Primary diagnoses included depressive disorders ( $50 \%$ of the sample), schizophrenia (25\%), and bipolar disorder (10\%). The remaining 15\% had diagnoses of anxiety disorders, personality disorders, and intellectual disabilities. The studies varied in their participant descriptions; most included maternal and child age and marital status; few provided further details (Table 1).

\section{Characteristics of studies}

Of the 23 papers, nine assessed mother-infant and maternal clinical outcomes, eight mother-infant outcomes, and six maternal clinical outcomes (Table 2). Five included a follow-up period, ranging from 1 to 6 years. Most used cohort designs, without control or comparison groups, although one presented a case series of two mothers. ${ }^{12}$ Five studies used a controlled design; four compared mothers admitted to an MBU with various groups, including healthy and depressed mothers in the community. One study compared different treatment programs. ${ }^{13}$

\section{Quality ratings}

QATSDD scores ranged from $29 \%$ to $83 \%$, with a mean of $62 \%$ (Table 2). Baker et al ${ }^{14}$ obtained the lowest rating, due to a lack of clarity of aims, justification of methodology, and description of procedure. In total, five papers scored as moderate quality, 12 as good, and six as high (Table 2). No relationship was observed between quality and design or year of publication. As this is the first review of MBU-related psychological outcomes, all studies were retained to present a comprehensive picture of the available research.

\section{Study findings}

Findings were grouped by outcome and methodology (see Table 2 for a summary).

\section{Maternal mental health outcomes}

Fifteen studies assessed maternal outcomes. Four used the Marcé checklist, an international scale using categories of "symptom-free", "considerably improved", "slightly improved", and "no change or decline". Ten used standardized tools, such as the Beck Depression Inventory (BDI)-II, ${ }^{15}$ but one ${ }^{16}$ was unclear about the method, appearing to use clinical judgment.

\section{The Marcé checklist}

The Marcé checklist is an international checklist to be completed with all joint admissions, with 53 items regarding diagnosis, the presence or absence of specific symptoms, treatment and outcome, and information on obstetric history and ethnicity. Two papers ${ }^{17,18}$ detailed Marcé audits in the UK, covering 1996-2002 and 1994-2000, respectively. A further two reported audits of French MBUs over 2001-2007 $7^{19}$ and $1999-2000 .^{20}$ To compare data categories, "symptom-free" and "considerably improved" were combined, as were the "no change" and "worse" categories The results were comparable across audits, with around $70 \%$ "symptom-free or considerably improved". Similar research assessed women admitted from 1959 to 1965; $44.6 \%$ were symptom-free at discharge, although $14.8 \%$ had poor adjustment at follow-up. ${ }^{16}$ Unfortunately, a mixture of methods were used to assess follow-up outcomes, making assessments imprecise, with small samples prohibiting valid conclusions. ${ }^{16}$ Furthermore, given the timeframe of this study, it is questionable how comparable these figures are to more recent research.

Maternal education, occupation, employment status, relationship status, and substance use during pregnancy were identified as predicting being "symptom-free or considerably improved". ${ }^{19}$ Women with personality disorders or nonaffective psychotic disorders improved less than women with mood or acute transient psychotic disorders. ${ }^{18,20}$ 
Table I Settings of studies included in review and reported demographic characteristics (in chronological order)

\begin{tabular}{|c|c|c|c|c|c|c|}
\hline $\begin{array}{l}\text { Paper } \\
\text { number }\end{array}$ & Author & Country & Unit size & Setting & Staff & Intervention \\
\hline$I^{\#}$ & $\begin{array}{l}\text { Meltzer-Brody } \\
\text { et } \mathrm{al}^{30}\end{array}$ & USA & $\begin{array}{l}5 \text { beds } \\
\text { No overnight } \\
\text { facility for } \\
\text { children }\end{array}$ & MBU & $\begin{array}{l}\text { Nurses, lactation consultants, } \\
\text { chaplain, recreational and } \\
\text { occupational therapists, } \\
\text { psychologists, psychiatrists, } \\
\text { social worker, yoga instructor }\end{array}$ & $\begin{array}{l}\text { Group and individual therapies: } \\
\text { art, relaxation, behavioral, and } \\
\text { mindfulness-based cognitive } \\
\text { therapy, M-wave biofeedback } \\
\text { therapy, mother-infant } \\
\text { attachment therapy, family and } \\
\text { partner-assisted interpersonal } \\
\text { psychotherapy, therapeutic yoga, } \\
\text { spiritual support }\end{array}$ \\
\hline 2 & Reddy et al ${ }^{12}$ & India & $*$ & MBU & * & $*$ \\
\hline 3 & Vliegen et $\mathrm{a}^{21}$ & Belgium & $\begin{array}{l}8 \text { beds } \\
4 \text { beds }\end{array}$ & 2 MBUs & $\begin{array}{l}\text { Psychiatrist, a child, and an adult } \\
\text { psychologist-psychotherapist, } \\
\text { psychomotor and creative } \\
\text { therapist, social worker, nurses, } \\
\text { child-care worker }\end{array}$ & $\begin{array}{l}\text { Systemic, psychodynamic, and } \\
\text { cognitive approaches to treat } \\
\text { mother and mother-infant } \\
\text { relationship }\end{array}$ \\
\hline $4^{\#}$ & Kenny et al $\left.\right|^{50}$ & UK & 13 beds & MBU & $\begin{array}{l}\text { Psychiatrist, psychologist, } \\
\text { nurses, OTs, social workers, } \\
\text { nursery nurses }\end{array}$ & $\begin{array}{l}\text { Medication, psychological } \\
\text { therapies, video interaction } \\
\text { work }\end{array}$ \\
\hline 5 & Bilszta et $\mathrm{al}^{13}$ & Australia & $*$ & 2 MBUs & Psychiatrist, nurses & $\begin{array}{l}\text { Practical baby-care sessions, } \\
\text { group discussions, CBT, music } \\
\text { therapy, art therapy, medication }\end{array}$ \\
\hline $6^{\#}$ & $\begin{array}{l}\text { Glangeaud- } \\
\text { Freudenthal } \\
\text { et al }{ }^{19}\end{array}$ & France & $*$ & $13 \mathrm{MBUs}$ & * & * \\
\hline $7^{\#}$ & Bergink et $\mathrm{al}^{37}$ & Netherlands & 5 beds & MBU & * & Medication \\
\hline $8^{\#}$ & Pawlby et al ${ }^{53}$ & UK & 12 beds & MBU & * & Video interaction feedback \\
\hline 9 & Vliegen et $\mathrm{a}^{25}$ & Belgium & $*$ & $2 \mathrm{MBUs}$ & * & $*$ \\
\hline 10 & $\begin{array}{l}\text { Noorlander } \\
\text { et al }{ }^{40}\end{array}$ & Netherlands & $*$ & MBU & * & $\begin{array}{l}\text { Medication, video intervention, } \\
\text { feedback from nursing staff, } \\
\text { therapy group }\end{array}$ \\
\hline
\end{tabular}




\begin{tabular}{|c|c|c|c|c|}
\hline $\begin{array}{l}\text { Maternal (M) and infant } \\
\text { (I) age (years and weeks/ } \\
\text { months, respectively) }\end{array}$ & Education/occupation & Marital status & Primiparous & Ethnicity \\
\hline $\begin{array}{l}\text { M: } 28.8 \text { years } \\
\text { I: } 16 \text { weeks }\end{array}$ & $*$ & $\begin{array}{l}49 \% \text { married } \\
12 \% \text { cohabiting } \\
23 \% \text { single } \\
6 \% \text { divorced } / \text { separated } \\
1 \% \text { widowed }\end{array}$ & $19 \%$ & $\begin{array}{l}50 \% \text { White } \\
29 \% \text { African-American } \\
12 \% \text { Hispanic } \\
1 \% \text { Asian American } \\
10 \% \text { other }\end{array}$ \\
\hline $\begin{array}{l}\text { M: * } \\
\text { I: } 6 \text { months, I year }\end{array}$ & $*$ & $*$ & $*$ & $*$ \\
\hline $\begin{array}{l}\text { M: } 29.39 \text { years } \\
\text { I: } 4.17 \text { months }\end{array}$ & I 3.68 years of education & $*$ & $*$ & $*$ \\
\hline M: 31.4 years MBU & $*$ & Single: $20.4 \% \mathrm{MBU}$ & $42.9 \% \mathrm{MBU}$ & White: $58.2 \%$ MBU \\
\hline 30.6 years community ill & & $20.9 \%$ community ill & $35.8 \%$ community ill & $43.3 \%$ community ill \\
\hline $\begin{array}{l}28.8 \text { years healthy } \\
\text { I: } 20.6 \text { weeks } \mathrm{MBU} \\
8.9 \text { weeks community ill } \\
22.3 \text { weeks healthy }\end{array}$ & & $19.0 \%$ healthy & $68.2 \%$ healthy & $54.5 \%$ healthy \\
\hline $\begin{array}{l}\text { M: }{ }^{*} \\
\text { I: } 5.8 \text { weeks }\end{array}$ & $*$ & $\begin{array}{l}92 \% \text { married or } \\
\text { cohabiting }\end{array}$ & $*$ & $\begin{array}{l}99 \% \text { English language, } \\
85 \% \text { born in Australia }\end{array}$ \\
\hline $\begin{array}{l}\text { M: } 3 \text { I years } \\
\text { I: } 9.6 \text { weeks }\end{array}$ & $\begin{array}{l}29.5 \% \text { training } \\
11.7 \% \text { unemployed } \\
13.1 \% \text { disability } \\
17.2 \% \text { sick leave } \\
13.6 \% \text { not in labor force } \\
15 \% \text { other } \\
35.1 \% \text { high school and over } \\
46.5 \% \text { secondary } \\
17.8 \% \text { primary or unknown }\end{array}$ & $65.5 \%$ cohabiting & $62.1 \%$ & $\begin{array}{l}\text { Country of birth: } \\
83.6 \% \text { France } \\
16.4 \% \text { other }\end{array}$ \\
\hline $\begin{array}{l}\text { M: } 31.9 \text { years } \\
\text { I: } *\end{array}$ & $\begin{array}{l}52.9 \% \text { postsecondary } \\
\text { education }\end{array}$ & $96.1 \%$ married & $78.4 \%$ & $88.2 \%$ dutch ethnicity \\
\hline $\begin{array}{l}\text { M: } 34.6 \text { years schizophrenia } \\
32.2 \text { years depression }\end{array}$ & $\begin{array}{l}\text { Professional/managerial: } \\
25 \% \text { schizophrenia }\end{array}$ & $\begin{array}{l}\text { Single: } 53.3 \% \\
\text { schizophrenia }\end{array}$ & $\begin{array}{l}33.3 \% \text { schizophrenia } \\
43.5 \% \text { depression }\end{array}$ & $\begin{array}{l}\text { White: } 20 \% \\
\text { schizophrenia }\end{array}$ \\
\hline $\begin{array}{l}29.0 \text { years mania } \\
30.5 \text { years controls }\end{array}$ & $\begin{array}{l}40 \% \text { depression } \\
40 \% \text { mania }\end{array}$ & $\begin{array}{l}4.3 \% \text { depression } \\
41.7 \% \text { mania }\end{array}$ & $\begin{array}{l}50 \% \text { mania } \\
56 \% \text { controls }\end{array}$ & $\begin{array}{l}69.6 \% \text { depression } \\
50 \% \text { mania }\end{array}$ \\
\hline $\begin{array}{l}\text { I: II.0 weeks schizophrenia } \\
\text { I } 2.2 \text { weeks depression } \\
7.0 \text { weeks mania } \\
\text { I } 2.0 \text { weeks controls }\end{array}$ & $31.7 \%$ control & 0 controls & & $100 \%$ controls \\
\hline $\begin{array}{l}\text { M: } 29.39 \text { years } \\
\text { I: } 4.17 \text { weeks }\end{array}$ & $*$ & $*$ & $*$ & $*$ \\
\hline $\begin{array}{l}\text { M: } 32.16 \text { years postpartum } \\
\text { depression } \\
31.73 \text { years postpartum psychosis } \\
\text { I: } 2.29 \text { months postpartum } \\
\text { depression } \\
0.99 \text { months postpartum psychosis }\end{array}$ & $\begin{array}{l}\text { Postpartum depression } \\
23.1 \% \text { primary } \\
53.9 \% \text { secondary } \\
23.1 \% \text { higher } \\
\text { Postpartum psychosis } \\
0 \% \text { primary } \\
58.3 \% \text { secondary } \\
41.7 \% \text { higher }\end{array}$ & $\begin{array}{l}\text { Married/cohabiting: } \\
84.6 \% \text { postpartum } \\
\text { depression } \\
100 \% \text { postpartum } \\
\text { psychosis }\end{array}$ & $\begin{array}{l}53.8 \% \text { postpartum } \\
\text { depression } \\
91.7 \% \text { postpartum } \\
\text { psychosis }\end{array}$ & $*$ \\
\hline
\end{tabular}

(Continued) 
Table I (Continued)

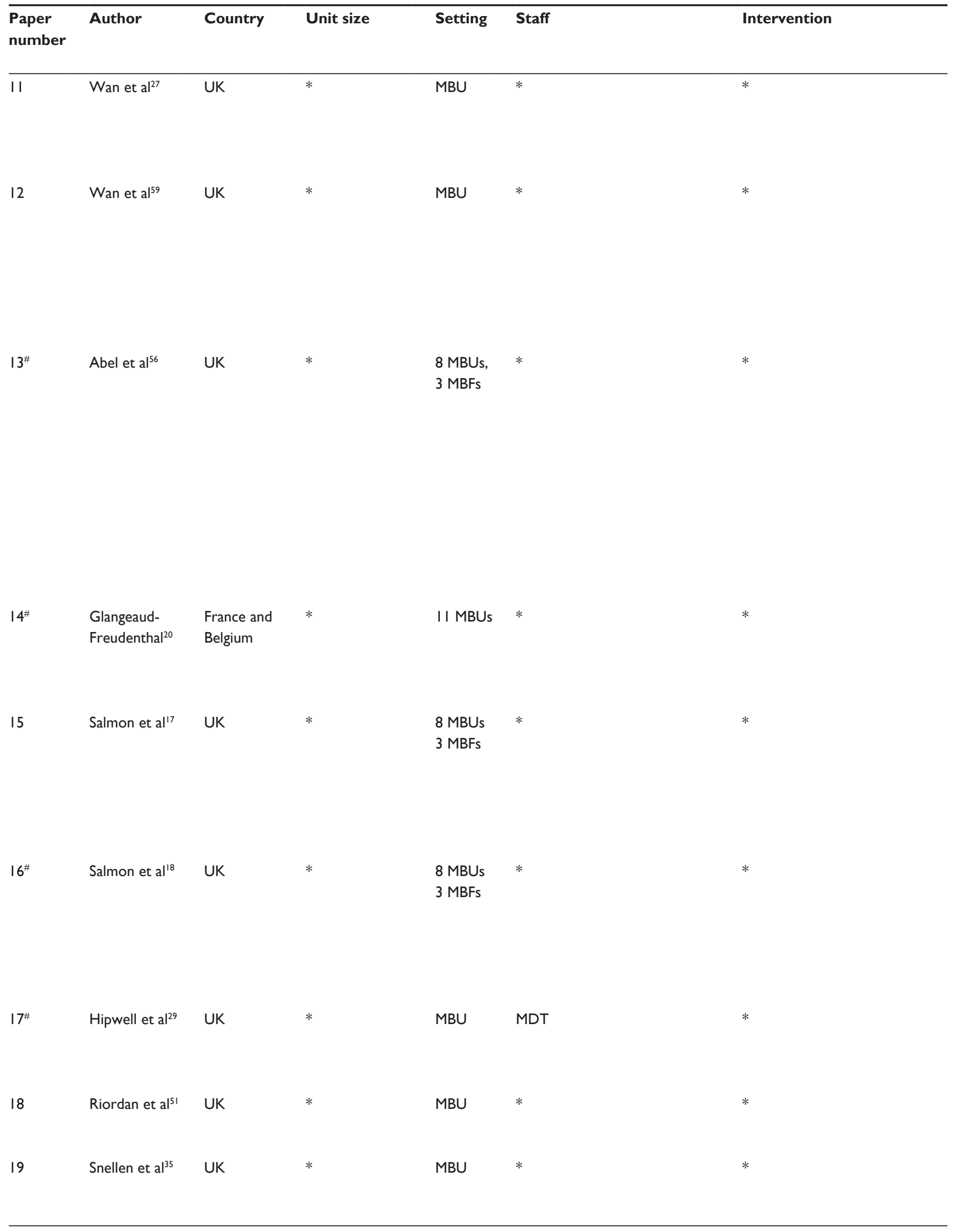




\begin{tabular}{|c|c|c|c|c|}
\hline $\begin{array}{l}\text { Maternal (M) and infant } \\
\text { (I) age (years and weeks/ } \\
\text { months, respectively) }\end{array}$ & Education/occupation & Marital status & Primiparous & Ethnicity \\
\hline $\begin{array}{l}\text { M: } 28.93 \text { years } \\
\text { I: } 8.27 \text { weeks }\end{array}$ & $\begin{array}{l}6 \text { professional/managerial } \\
4 \text { skilled/manual } \\
2 \text { semiskilled/unskilled } \\
3 \text { unemployed } \\
\text { I not classified }\end{array}$ & $87 \%$ married & $*$ & $*$ \\
\hline $\begin{array}{l}\text { M: } 30.54 \text { years schizophrenia } \\
27.43 \text { years bipolar disorder } \\
27.45 \text { years depression } \\
\text { I: * }\end{array}$ & $\begin{array}{l}\text { Professional to skilled manual: } \\
23 \% \text { schizophrenia } \\
57 \% \text { bipolar disorder } \\
27 \% \text { depressive illness } \\
\text { Educational qualification: } \\
54 \% \text { schizophrenia } \\
29 \% \text { bipolar disorder } \\
55 \% \text { depressive illness }\end{array}$ & $\begin{array}{l}\text { Married/cohabiting: } \\
\text { 39\% schizophrenia } \\
71 \% \text { bipolar disorder } \\
64 \% \text { depressive illness }\end{array}$ & $\begin{array}{l}69 \% \text { schizophrenia } \\
57 \% \text { bipolar disorder } \\
54 \% \text { depressive illness }\end{array}$ & $\begin{array}{l}\text { White: } 62 \% \\
\text { schizophrenia } \\
93 \% \text { bipolar disorder } \\
100 \% \text { depressive } \\
\text { illness }\end{array}$ \\
\hline $\begin{array}{l}\text { M: schizophrenia } \\
20 \%<20 \text { years, } 29 \% 25-29 \text { years, } \\
30 \% 30-34 \text { years, } 22 \% 35+\text { years } \\
\text { Affective disorder } \\
20 \%<20 \text { years, } 29 \% 25-29 \text { years, } \\
29 \% 30-34 \text { years, } 23 \% 35+\text { years } \\
\text { I: } 41 \%<3 \text { weeks schizophrenia } \\
22 \%<3 \text { weeks affective disorder }\end{array}$ & $\begin{array}{l}\text { Schizophrenia: } \\
28 \% \text { professional/managerial } \\
54 \% \text { semiskilled/unskilled } \\
18 \% \text { never employed } \\
\text { Affective disorder: } \\
51 \% \text { professional/managerial } \\
40 \% \text { semiskilled/unskilled } \\
9 \% \text { never employed }\end{array}$ & $\begin{array}{l}\text { Married/cohabiting: } \\
53 \% \text { schizophrenia } \\
76 \% \text { affective disorder }\end{array}$ & $\begin{array}{l}57 \% \text { schizophrenia } \\
56 \% \text { affective disorder }\end{array}$ & $\begin{array}{l}\text { Schizophrenia: } 56 \% \\
\text { White } \\
27 \% \text { Black African } \\
\text { or Caribbean } \\
\text { I1\% South Asian } \\
6 \% \text { other } \\
\text { Affective disorder: } \\
78 \% \text { White } \\
9 \% \text { Black African } \\
\text { or Caribbean } \\
7 \% \text { South Asian } \\
6 \% \text { other }\end{array}$ \\
\hline $\begin{array}{l}\text { M: } 30 \text { years } \\
\text { I: } 10.6 \text { weeks }\end{array}$ & $\begin{array}{l}29 \% \text { professional } \\
24 \% \text { unemployed } \\
22 \% \text { student } \\
14 \% \text { disability } \\
\text { II\% other }\end{array}$ & $76 \%$ living with partner & $*$ & $*$ \\
\hline $\begin{array}{l}\text { M: } 26 \% \text { I } 6-25 \text { years } \\
73 \% 26-50 \text { years } \\
\text { I: } *\end{array}$ & $\begin{array}{l}23 \% \text { professional/managerial } \\
18 \% \text { skilled manual } \\
42 \% \text { semiskilled/unskilled } \\
4 \% \text { unclassified } \\
7 \% \text { unemployed }\end{array}$ & $\begin{array}{l}68 \% \text { married/cohabiting } \\
5 \% \text { separated/divorced } \\
24 \% \text { single } \\
0.2 \% \text { widowed } \\
1 \% \text { other }\end{array}$ & $*$ & $\begin{array}{l}\text { II\% Black African } \\
6 \% \text { Black Caribbean } \\
8 \% \text { Indian/Pakistani/ } \\
\text { Bangladeshi } \\
1 \% \text { Chinese } \\
66 \% \text { White } \\
7 \% \text { other }\end{array}$ \\
\hline $\begin{array}{l}\text { M: } 26 \% 16-25 \text { years } \\
73 \% 26-50 \text { years } \\
\text { I: } *\end{array}$ & $\begin{array}{l}23 \% \text { professional/ } \\
\text { managerial } \\
18 \% \text { skilled manual } \\
42 \% \text { semiskilled/unskilled } \\
4 \% \text { unclassified } \\
7 \% \text { unemployed }\end{array}$ & $\begin{array}{l}68 \% \text { married/cohabiting } \\
5 \% \text { separated/divorced } \\
24 \% \text { single } \\
0.2 \% \text { widowed } \\
1 \% \text { other }\end{array}$ & $*$ & $\begin{array}{l}\text { II\% Black African } \\
6 \% \text { Black Caribbean } \\
8 \% \text { Indian/Pakistani/ } \\
\text { Bangladeshi } \\
1 \% \text { Chinese } \\
66 \% \text { White } \\
7 \% \text { other }\end{array}$ \\
\hline $\begin{array}{l}\text { M: } 29.5 \text { years } \mathrm{MBU} \\
28.9 \text { years control } \\
\text { I: } 4.1 \text { weeks } \mathrm{MBU} \\
\text { I.7 weeks control }\end{array}$ & $\begin{array}{l}\text { Nonmanual: } \\
60 \% \text { MBU } \\
63 \% \text { control }\end{array}$ & $\begin{array}{l}\text { Married/cohabiting: } \\
80 \% \mathrm{MBU} \\
88 \% \text { control }\end{array}$ & $\begin{array}{l}\text { MBU } 64 \% \\
\text { Control } 56 \%\end{array}$ & $*$ \\
\hline $\begin{array}{l}\text { M: } 3 \text { I } .5 \text { years schizophrenia } \\
25.7 \text { years affective disorder } \\
\text { I: } *\end{array}$ & $*$ & * & $*$ & $*$ \\
\hline $\begin{array}{l}\text { M: } 28.6 \text { years } \\
\text { I: } 16.9 \text { weeks }\end{array}$ & $*$ & $\begin{array}{l}53 \% \text { single } \\
27 \% \text { married } \\
20 \% \text { de facto }\end{array}$ & $*$ & $*$ \\
\hline
\end{tabular}


Table I (Continued)

\begin{tabular}{|c|c|c|c|c|c|c|}
\hline $\begin{array}{l}\text { Paper } \\
\text { number }\end{array}$ & Author & Country & Unit size & Setting & Staff & Intervention \\
\hline 20 & Milgrom et al ${ }^{57}$ & Australia & $\begin{array}{l}6 \text { beds, focus } \\
\text { on mothers } \\
\text { with psychosis }\end{array}$ & $\mathrm{MBU}$ & $\begin{array}{l}\text { Consultant psychiatrist, } \\
\text { psychiatric nurses, pediatrician, } \\
\text { psychologist, maternal and } \\
\text { child health nurse, social } \\
\text { worker, occupational therapist, } \\
\text { psychiatry registrar }\end{array}$ & $\begin{array}{l}\text { Medication, nursing, mothering } \\
\text { skills, baby play group, CBT, } \\
\text { family/couples work, social skills } \\
\text { training, relaxation, daily living } \\
\text { skills }\end{array}$ \\
\hline $21^{\#}$ & $\begin{array}{l}\text { Hipwell and } \\
\text { Kumar }^{44}\end{array}$ & UK & $*$ & $\mathrm{MBU}$ & MDT & * \\
\hline 22 & Bardon et a $\left.\right|^{16}$ & UK & 10 beds & $\mathrm{MBU}$ & Nurses & $\begin{array}{l}\text { Medication, weekly group } \\
\text { therapy, staff discussion, } \\
\text { individual psychotherapy, OT }\end{array}$ \\
\hline $23^{\#}$ & Baker et al ${ }^{14}$ & UK & $\begin{array}{l}8 \text { beds, focus } \\
\text { on mothers } \\
\text { with psychosis }\end{array}$ & $\mathrm{MBU}$ & $\begin{array}{l}\text { "Mentally trained nurses", } \\
\text { nursery nurses, psychiatrist, } \\
\text { psychologist, social worker }\end{array}$ & $\begin{array}{l}\text { ECT, chlorpromazine, } \\
\text { atmosphere of emotional } \\
\text { warmth and support }\end{array}$ \\
\hline
\end{tabular}

Notes: *Information not provided; ${ }^{*}$ external funding sources.

Abbreviations: MBU, mother and baby unit; OT, occupational therapist; CBT, cognitive behavioral therapy; MBF, mother and baby facility; MDT, multidisciplinary team; $E C T$, electroconvulsive therapy.

\section{Standardized psychometrics}

Several studies used psychometric self-report tools to measure change during follow-up periods. One ${ }^{21}$ showed significant decreases in depression (BDI-II), ${ }^{15}$ state and trait anxiety (State Trait Anxiety Inventory) ${ }^{22}$ and state anger (State Trait Anger Expression Inventory). ${ }^{23}$ Furthermore, participants reported less negative and more positive affect (Leuven Emotions Scale). ${ }^{24}$ Therefore, the majority had good mental health at follow-up, although 39\% were clinically depressed. Further analysis ${ }^{25}$ noted participants remained depressed if they were more severely depressed and more self-critical (Depressive Experiences Questionnaire) ${ }^{26}$ at admission. Another study ${ }^{27}$ assessed depression (BDI) ${ }^{28}$ over a 4- to 6-year-follow-up period, with comparable findings: $21 \%$ of participants were clinically depressed at follow-up, and $60 \%$ reported current mental health needs.

In research reporting slightly better outcomes, ${ }^{29} 16 \%$ of MBU participants reported some depressive symptoms at 12 months postpartum, although none met diagnostic criteria (BDI) ${ }^{28}$ In contrast, $44 \%$ of a community ill group reported symptoms and $19 \%$ met diagnostic criteria. This may indicate the superiority of inpatient treatment or reflect intergroup differences. The community ill group had diagnoses of depression, whereas the MBU group had other diagnoses, including schizophrenia. Therefore, outcome differences could be due to variations in the chronicity or treatability of each disorder. Additionally, follow-up was conducted at 12 months postpartum rather than a specified time since MBU discharge. Therefore, it is questionable whether this could be viewed as MBU outcome data.

These results are consistent with research using psychometric tools to assess change over admission. One study $^{30}$ found depression and anxiety significantly reduced (Edinburgh Postnatal Depression Scale [EPDS], Patient Health Questionnaire 9, Generalized Anxiety Disorder scale 7), ${ }^{31-33}$ and participants reported an increase in overall functioning (Work and Social Adjustment Scale). ${ }^{34}$ Similar research ${ }^{35}$ with participants with schizophrenia recorded significant improvements in psychotic symptoms (Positive and Negative Syndrome Scale, PANSS). ${ }^{36}$ Focusing on postpartum psychosis, a further study ${ }^{37}$ showed $92.2 \%$ of participants were symptom-free at discharge (EPDS, Clinical Global Impression scale [CGI], Young Mania Rating Scale), $, 31,38,39$ although those with depressive features had a longer recovery period than those with manic features.

One study ${ }^{13}$ used the EPDS ${ }^{31}$ to assess video-feedback interventions during admission, comparing this to verbal feedback and standard care. EPDS scores significantly improved across groups, and no intervention was significantly superior to any other.

Two studies reported changes in illness severity over admission, although neither statistically assessed these for 


\begin{tabular}{|c|c|c|c|c|}
\hline $\begin{array}{l}\text { Maternal }(M) \text { and infant } \\
\text { (I) age (years and weeks/ } \\
\text { months, respectively) }\end{array}$ & Education/occupation & Marital status & Primiparous & Ethnicity \\
\hline M: 28.8 years & I4\% employed & $39 \%$ single & $58 \%$ & $*$ \\
\hline \multirow[t]{3}{*}{ I: 3.8 months } & $25 \%$ home duties & $44 \%$ married & & \\
\hline & $61 \%$ unemployed and & $14 \%$ de facto & & \\
\hline & receiving benefits & $3 \%$ separated & & \\
\hline M: 29.7 years unipolar depression & Nonmanual: & Married/cohabiting: & $54 \%$ unipolar depression & $*$ \\
\hline 28.2 years bipolar depression & $68 \%$ unipolar depression & $82 \%$ unipolar & $69 \%$ bipolar depression & \\
\hline 26.8 years schizophrenia & $54 \%$ bipolar depression & depression & $53 \%$ schizophrenia & \\
\hline I: 6.9 weeks unipolar depression & $27 \%$ schizophrenia & $77 \%$ bipolar depression & & \\
\hline 4.3 weeks bipolar depression & & $40 \%$ schizophrenia & & \\
\hline \multicolumn{5}{|l|}{ 5.I weeks schizophrenia } \\
\hline M: 28.3 years & $*$ & $*$ & $53 \%$ & $*$ \\
\hline \multicolumn{5}{|l|}{ I: I 3 weeks } \\
\hline M: 26.6 years $\mathrm{MBU}$ & $*$ & * & $60 \% \mathrm{MBU}$ & $*$ \\
\hline 27.4 years control & & & $65 \%$ control & \\
\hline I: $*$ & & & & \\
\hline
\end{tabular}

significance. Firstly, a decrease in $\mathrm{CGI}^{38}$ scores was noted for those with both postpartum depression and postpartum psychosis. ${ }^{40}$ Secondly, lower Wittenborn Psychiatric Rating Scale ${ }^{41}$ scores were found for participants admitted with their child than those without. ${ }^{14}$ Without statistical assessment, it is hard to draw conclusions from these differences.

Therefore various psychometric tools have demonstrated improvements in maternal mental health symptoms over admission and into follow-up periods, with slower recovery for those with depressive feature ${ }^{37}$ and self-criticism. ${ }^{25}$ While some superiority has been demonstrated to community-based treatment, the research also shows symptoms persisting at follow-up.

\section{Summary of maternal mental health outcomes}

Numerous data-collection methods, follow-up periods, and inclusion criteria were used, yet the results indicate improvement in maternal mental state from admission to discharge or to follow-up. These findings are promising, as are the results of the studies using control groups. However, the methodological concerns, including design issues, such as small sample sizes and diagnostic group differences, limit the implications of these results. Diagnostically, it appears that psychotic disorders, personality disorders, and high levels of self-criticism are related to poorer outcomes, but there is not enough research to draw firm conclusions.

\section{Mother-infant outcomes}

Seventeen papers assessed child or mother-infant outcomes (Table 2), using the Marcé checklist ( $n=4)$ or other standardized tools $(n=13)$.

\section{Rating scales of the mother-infant relationship}

Several studies used scales to assess the mother-infant relationship, including either self-report or staff-rated scales. The Emotional Availability - Self-Report scale identified significant increases in the child's capacity to involve their parent and the affect quality of the interaction over a 3.5-year follow-up period. ${ }^{21}$ Participants who were depressed at follow-up had significantly lower levels of mutual attunement than nondepressed participants.

Similar progress was recorded over admission with both a staff-completed scale (Bethlem Mother-Infant Interaction Scale [BMIS]) $)^{42}$ and self-report scale (Postpartum Bonding Questionnaire). ${ }^{40,43}$ At admission, participants with postpartum depression rated their bonding as more problematic than participants with postpartum psychosis. In contrast, staff ratings recorded more difficulties for participants with postpartum psychosis. At discharge, all self-report scores were below clinical thresholds and staff ratings were reduced. A further study also showed BMIS scores improved significantly over MBU admission, and differed by diagnosis; scores at discharge were better for 

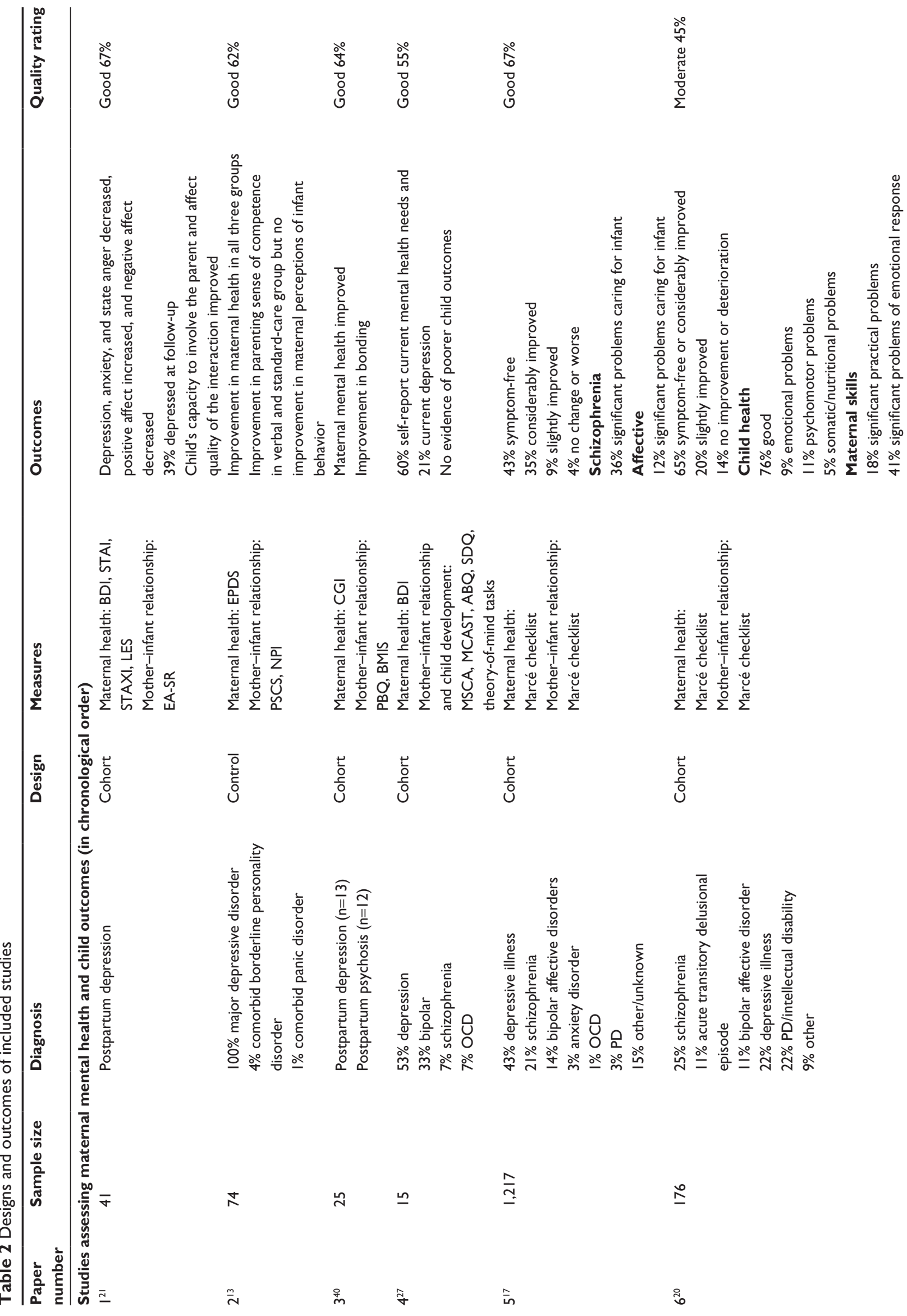


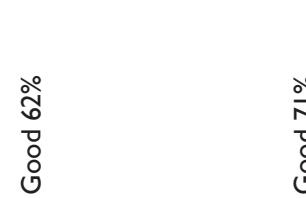

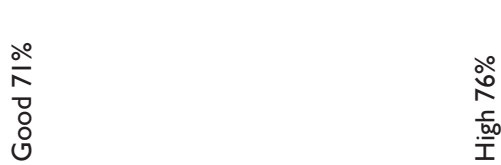

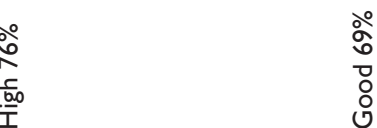

के
$\stackrel{\circ}{0}$
$\stackrel{0}{\circ}$
$\stackrel{0}{0}$

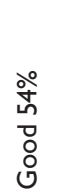
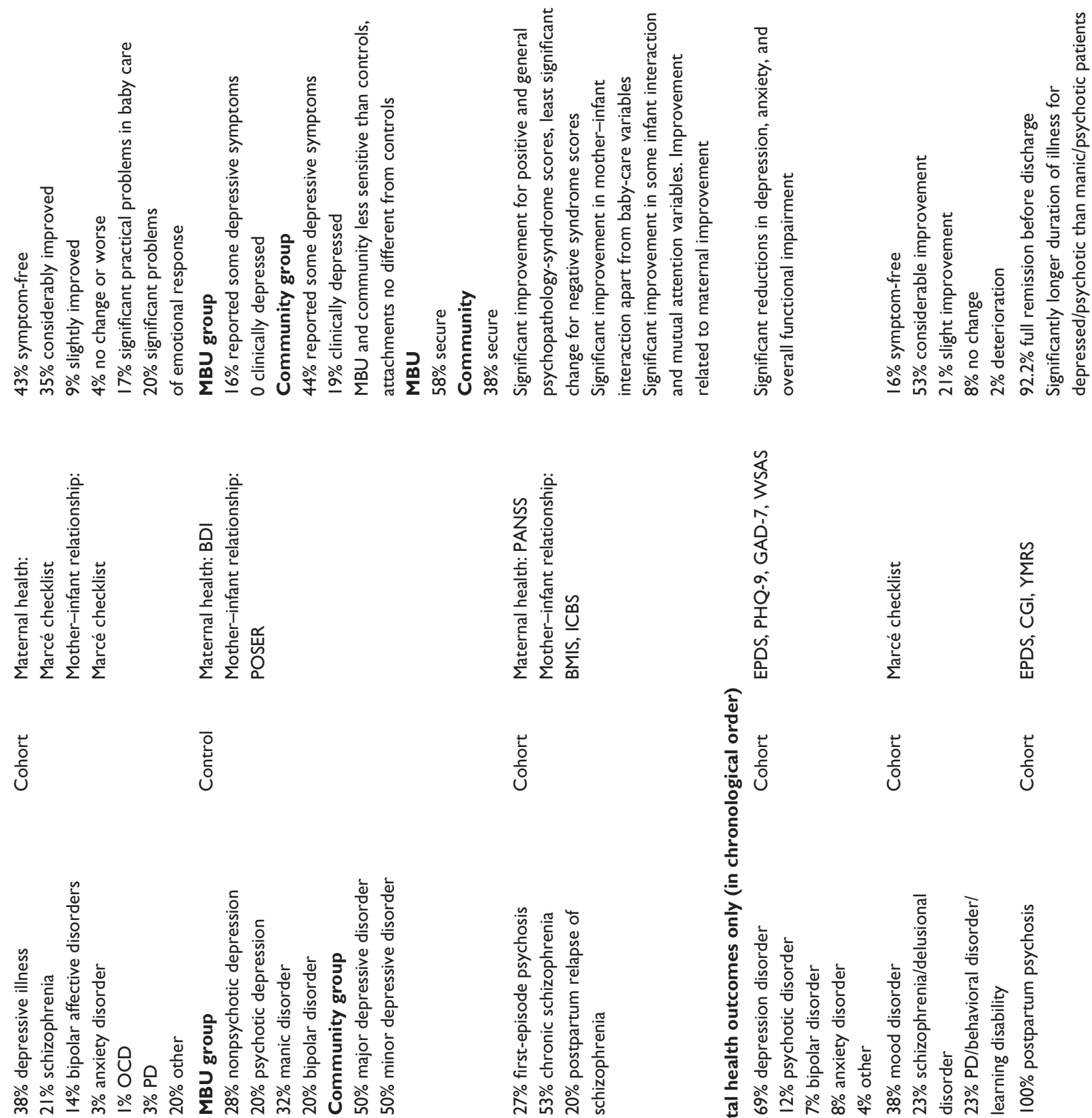

$\frac{5}{0}$

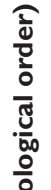

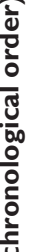
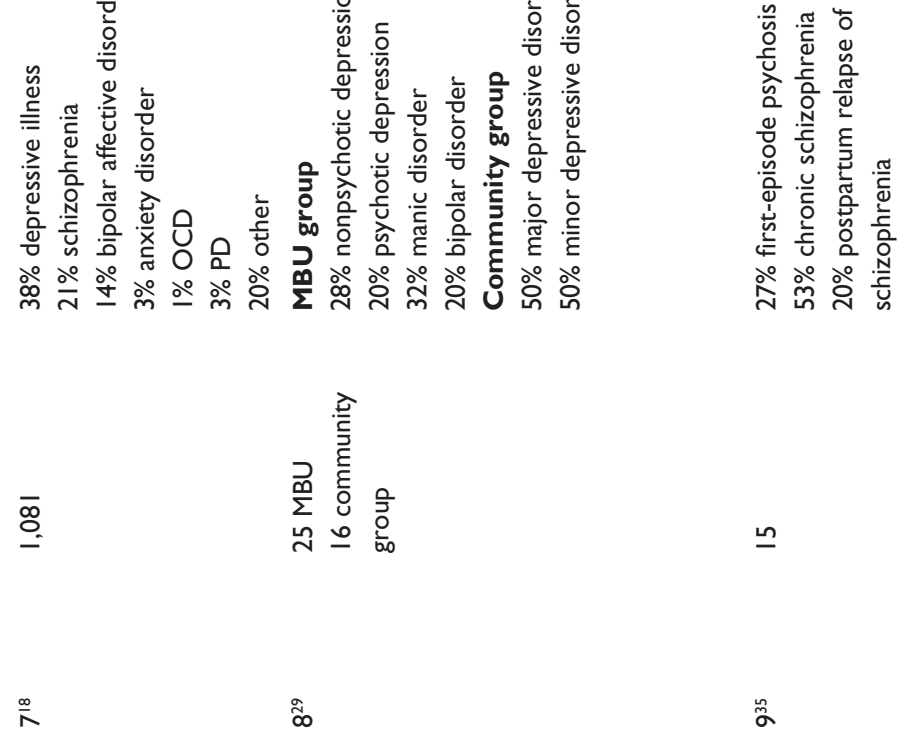

느

骂

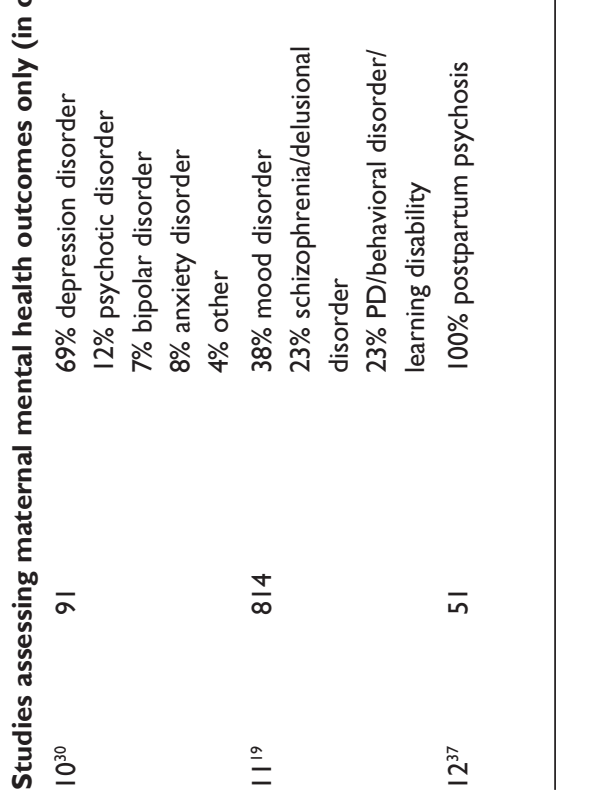




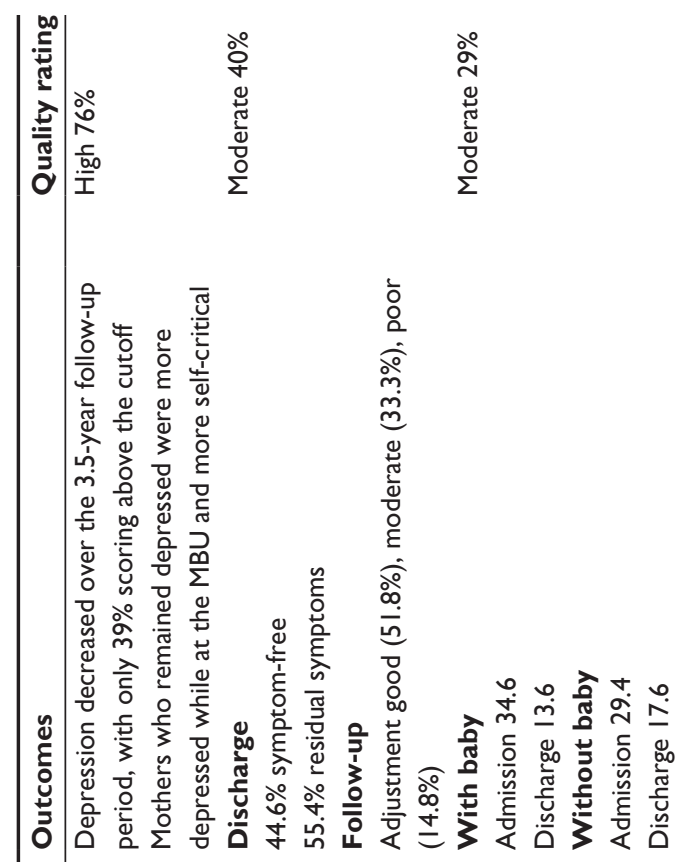

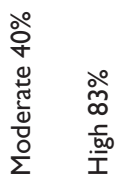
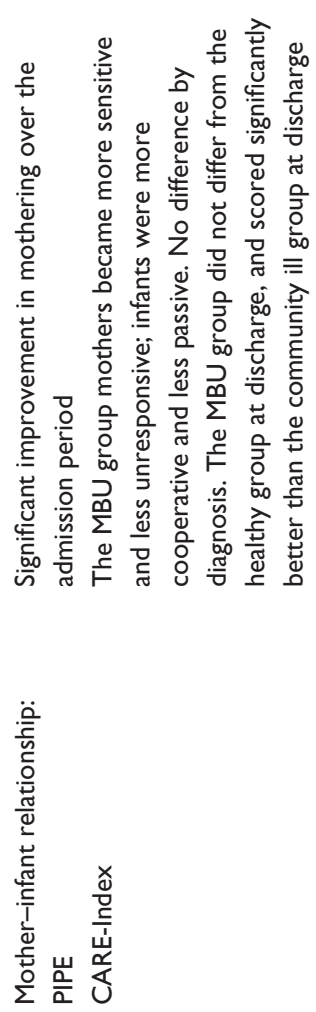

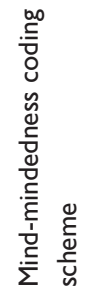

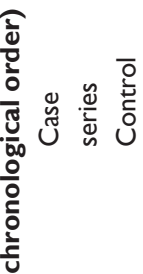

نั

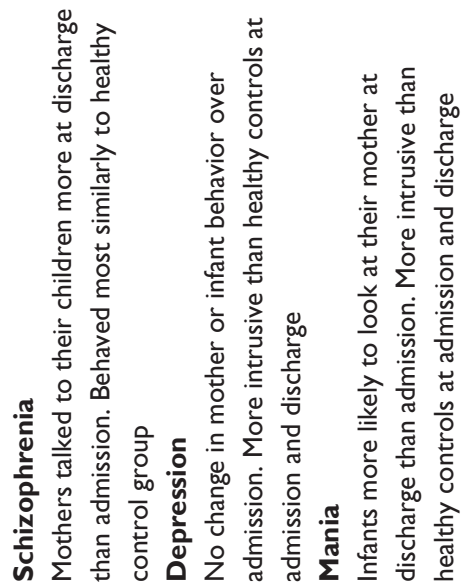

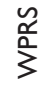

훙

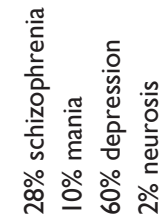

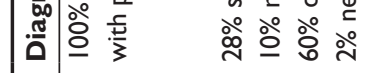

ชิ

$\frac{0}{n}$
$\frac{n}{0}$
$\frac{0}{0}$
ติ

产

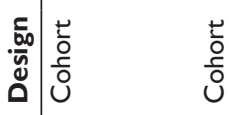

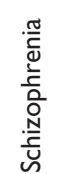

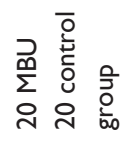

는

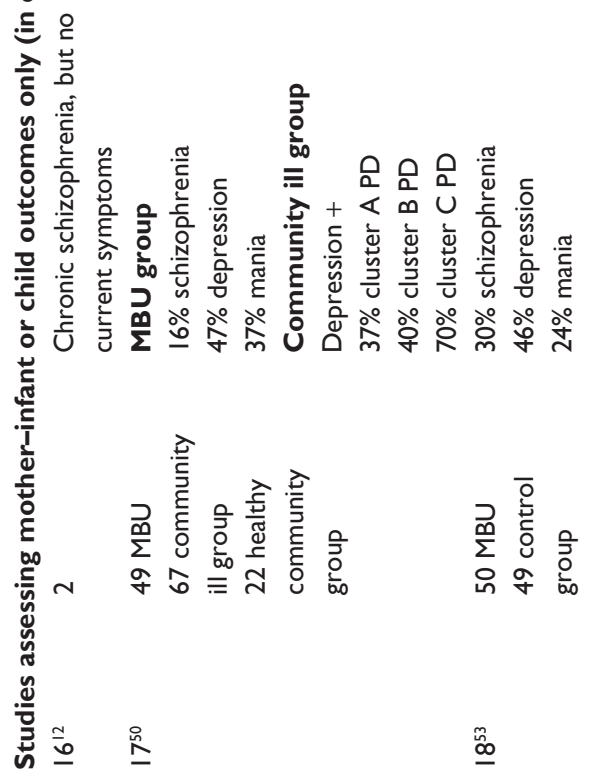



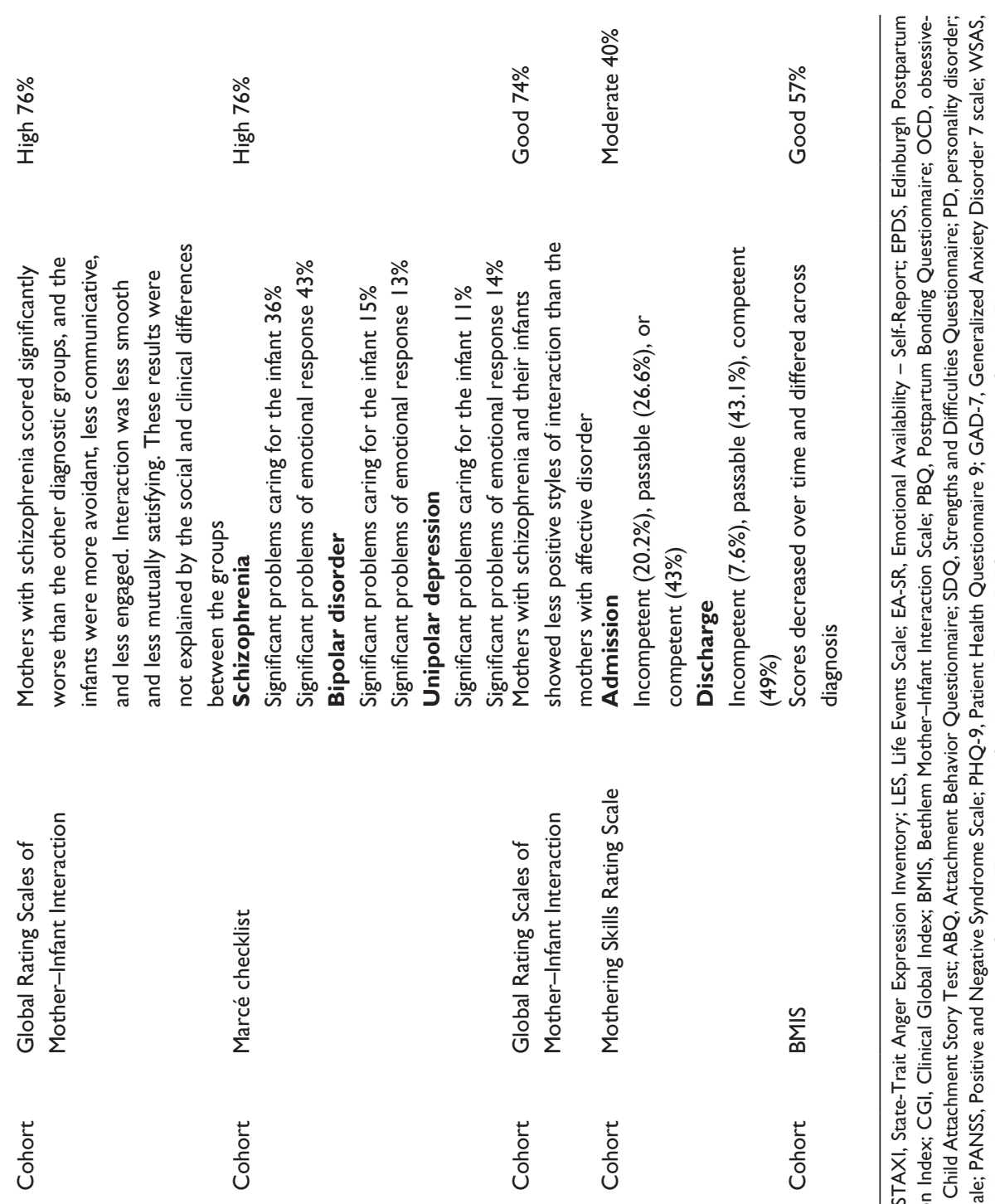

品

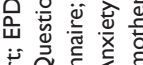

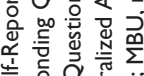

g

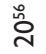

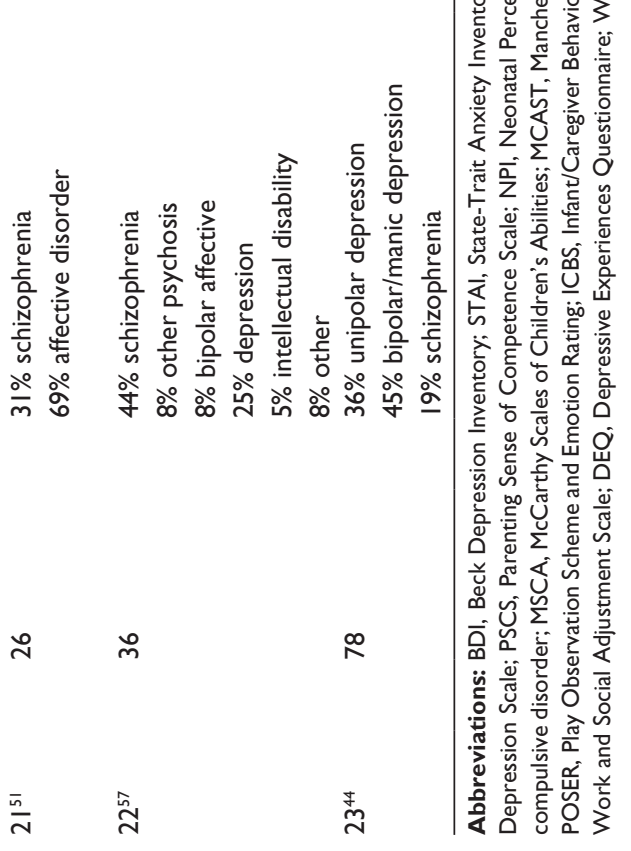


those with unipolar depression or bipolar disorder than those with schizophrenia. ${ }^{44}$ In a sample of mothers with schizophrenia, ${ }^{35}$ scores increased on most BMIS subscales over admission. The Infant/Caregiver Behavior Scale recorded significant improvements on dyadic and maternal responses, but only on three of eight infant-response scales. ${ }^{35}$ However, validity or reliability Infant/Caregiver Behavior Scale data were unavailable, so these findings must be viewed cautiously.

In a comparison of three groups of participants receiving video feedback, verbal feedback, or standard care, improved parenting confidence (Parenting Sense of Competence Scale) $)^{45}$ was noted for the verbal feedback and standard-care groups, but perceptions of infant behavior remained unchanged for all three groups (Neonatal Perception Index). ${ }^{13,46}$ Although data were not presented, the authors ${ }^{13}$ reported no superiority of either intervention over standard care, nor any significant difference between the interventions.

In summary, rating scales indicated improvements in the mother-infant relationship through admission and into follow-up. They suggested that mothers with depression perceive more difficulties in their relationship than are recorded by nursing staff. There is some indication that participants with schizophrenia have more difficulties in the mother-infant relationship at discharge than those with other diagnoses. Improvements in the relationship were not shown to differ according to the offered intervention.

\section{Observational measures of the mother-infant relationship}

A range of observational measures was used to assess the mother-infant relationship at either discharge or follow-up. At 12 months postpartum, 58\% of children of MBU-admitted participants were securely attached, as were $38 \%$ of children of community participants, neither differing significantly from their matched controls. ${ }^{29}$ This is markedly similar to rates of secure attachment at 4-6 years after discharge from an MBU. ${ }^{27}$ Higher rates of insecure attachments were noted for MBU participants with affective disorders compared to those with bipolar disorders, ${ }^{29}$ consistent with the literature on the different psychopathologies. ${ }^{47}$ The depressed MBU participants engaged in significantly less affectionate talk with their children than the depressed community group (Play Observation Scheme and Emotion Rating). ${ }^{29,48}$

In contrast, when compared with community-ill participants, the CARE-Index ${ }^{49}$ showed MBU participants had significantly better mother-infant relationships. ${ }^{50}$ However, all community ill participants had comorbid personality disorders, compared with only one MBU participant. Previous research related personality disorders to poorer treatment outcomes, ${ }^{19}$ suggesting improvements in the MBU participants could be due to diagnostic differences. This could also explain the contradiction with the previous research, ${ }^{29}$ which was limited to participants with depression..$^{29}$ Furthermore, postpartum assessment times varied greatly ${ }^{50}$ (8.9 weeks for the community ill group, 22.3 weeks for the healthy group, and 20.6 weeks for the MBU group), meaning the communityill group had less time to adjust to motherhood.

Two studies ${ }^{51,59}$ used the Global Rating Scales of MotherInfant Interaction ${ }^{52}$ to assess participants at MBU discharge. Again, results differed by diagnosis: participants with schizophrenia had the lowest interaction scores, being significantly less accepting and warm than participants with affective disorders. Ratings of video interactions of MBU participants and healthy mothers were used to focus on mind-mindedness or the caregivers' ability to understand their child's internal state. ${ }^{53,54}$ MBU participants with schizophrenia talked to their infants significantly more, and infants of participants with mania looked at their mothers more at discharge than admission. However, the research failed to reveal the hypothesized intergroup difficulties in mind-mindedness; the authors related this finding to the assessment schedule, which was designed for healthy mothers and did not appear to capture observed instances of nonattunement.

Outcomes from video feedback were reported in a case series of two symptom-free participants with schizophrenia admitted to the same MBU in India, ${ }^{12}$ and both showed improvements on the Pediatric Infant Parent Exam; ${ }^{55}$ however, the sample was too small to draw meaningful conclusions.

Therefore, the observational measures recorded similar findings to the rating scales, revealing improvements in mother-infant interactions over admission and into follow-up. Regarding diagnosis, the research is mixed. More difficulties appeared to be experienced at discharge by participants with schizophrenia or personality disorders, although attachment styles at follow-up were less positive for participants with affective disorders.

\section{Parenting skills}

Three studies used the Marcé checklist to report on parenting skills. ${ }^{17,20,56}$ The French audit ${ }^{20}$ recorded more parenting difficulties than either UK audit. ${ }^{17,56}$ When viewed by diagnosis, outcomes were significantly worse for those with schizophrenia; however, schizophrenia rates in the French audit ${ }^{20}$ were nearly double those in either UK audit, ${ }^{17,56}$ potentially explaining this discrepancy.

The Mothering Skills Rating Scale was used to assess aspects of parenting skills, including practical management, 
daily routine, and interactional tasks, which were rated "incompetent", "passable" or "competent". ${ }^{57}$ Data were missing for 14 of the 36 participants, and no analysis contrasting those with complete data and those with missing data was reported. Potentially, staff made more effort to complete ratings for specific mothers, introducing bias. A trend for improvement in parenting competence over admission was observed, as "incompetent" ratings decreased and "passable" ratings increased. Unfortunately, it is difficult to differentiate this from the natural increase in parenting skills that might be expected for all mothers.

\section{Child development}

Only two studies considered the development of children whose mothers were admitted to an MBU. One ${ }^{20}$ included the Marcé checklist data of child's health at discharge: $76 \%$ of children were rated as having good health or no problems, and recorded difficulties were often transitory. A further study ${ }^{27}$ used a range of standardized tools with children whose mothers had been discharged 4-6 years previously. No concerns with behavioral, emotional, or cognitive functioning were revealed. However, only $28 \%$ of eligible mothers participated, limiting the power of this study.

\section{Summary of mother-infant outcomes}

The quality of mother-infant interactions generally improved, regardless of assessment method. Although mothers with schizophrenia had the poorest outcomes, attachment studies suggest higher levels of insecure attachment in children of mothers with affective disorders.

\section{Discussion}

This review systematically examined 23 identified studies reporting on outcomes following MBU admission. In summary, the research indicated improvements in maternal mental health, mother-infant relationship, and child development. However, studies also indicated poorer outcomes for specific groups of mothers, particularly those with a personality or psychotic disorder, severe depression, or high self-criticism. Furthermore, outcomes were influenced by variables indicating socioeconomic status, including education and employment. However, without further research, it remains possible that the diagnostic differences were related to the higher rates of stigma attached to specific diagnoses. ${ }^{58}$

The second aim of this review concerned the methodologies used: given the urgent care MBUs provide, it was not surprising that no randomized control trials were identified. Although only four studies included a control group, these were not always optimal, eg, differing substantially from the MBU participants in diagnosis. Therefore, sample sizes, outcome measures, and designs varied greatly, making it difficult to draw overarching conclusions, yet reflecting the reality of clinical services. Most studies appropriately assessed participants at admission and discharge only, but without follow-ups to assess lasting change.

With regard to the measures commonly used, there was little consistency across the studies. Maternal mental health was assessed using self-report or staff-rating scales, mostly the BDI $(n=4)$ for symptom change and the Marcé checklist $(n=4)$ for improvement indicators. Specific symptoms or difficulties, such as psychosis, were assessed with specific measures, such as the PANSS. The mother-infant relationship was assessed using staff rating, video-observation rating, and self-report scales. Child development was assessed using self-report, observational measures, and the Marcé checklist. Overall, no single outcome measure or methodology dominated across the 23 studies.

This review also aimed to report on the efficacy of MBUbased interventions; however, no intervention appeared to be consistently offered, nor was any superior when compared with other interventions. For example, video feedback was used, but was not found to be superior to verbal feedback or standard care. ${ }^{13}$ An important observation was that most studies provided limited descriptions of the staffing, theoretical underpinnings, and psychosocial interventions provided in each unit. Therefore, it was not possible to explore relationships between these interventions and outcomes.

\section{Limitations}

A major limitation is the variable quality of the available studies. While randomized controlled trials were not expected, even the studies with control groups had weaknesses, limiting the strength of the conclusions that can be drawn. Furthermore, descriptions of each MBU and their participants were sparse. Although the use of the QATSDD quality measure allowed for the diverse designs used in these 23 papers to be compared, there were methodological weaknesses that it did not capture, including the suitability of control-group participants and differing assessment time points. Additionally, scores were categorized by the authors into groups to assist with interpretation, so must be viewed with some caution. However, rather than using opportunistic control groups, researchers should seek more appropriate comparators and include follow-ups, as patients may access additional services after MBU discharge.

\section{Recommendations for future research}

This review highlighted the limited quality of existing research. Future studies should at the very least include 
detailed descriptions of the studied unit, including size, staffing, and intervention approaches, allowing units to be contrasted and outcomes compared. Socioeconomic details should also be reported, given their possible association with outcome. Greater use of control groups would provide a comparative base for identified outcomes; ideally, these would be matched in terms of child age and socioeconomic or educational background. However, outside the research context, the use of control groups as comparators may not be practicable, which is why greater priority should be given to the systematic reporting of routinely collected admission and discharge data. Currently, the range of measures being used makes it difficult to compare outcomes reported by MBUs. Although the Marcé checklist was used in some studies, it is not a validated assessment tool sensitive to change. The BMIS was used to assess the mother-infant relationship in three studies. However, many observational measures (including the CARE-Index) can only be used if staff are trained in their use, are reliable raters, and receive regular supervision to ensure interrater reliability remains high, which can be costly undertakings for services. Similarly, assessing maternal mental health can be complex; measures may be more or less relevant depending on the diagnostic group. At the very least, these outcomes should be assessed at admission and discharge, and where possible with an appropriate follow-up.

\section{Recommendations for clinical practice}

In clinical practice, measures need to be quick, easy, and require minimal training. For maternal mental health, staffrated $\mathrm{CGI}^{38}$ scales of illness severity, improvement, and intervention effect may be appropriate, and could be completed during ward round. Quality will depend on the expertise and experience of staff completing the rating; however, this very quick measure is freely available, requires no training, and is transdiagnostic. Additionally, the BMIS, developed in MBUs for nurses to rate the mother-infant relationship, is freely available and requires no training. At admission, some patients may lack the capacity to complete measures; therefore, staff-rated measures may be more appropriate. However, it could be informative to collect self-report measures when possible, given the differences revealed by research comparing these methods. ${ }^{40}$ If similar measures are used, this would allow for comparisons with other MBUs and their clinical service contexts. Furthermore, the data relating to specific diagnostic groups and socioeconomic characteristics suggest staff may be able to identify mothers requiring more support, allowing for the planning of longer admissions or longer outpatient follow-up.

\section{Conclusion}

Joint admissions were recommended by Main ${ }^{4}$ in 1958, and specialist MBUs followed shortly thereafter. Despite this history, this is the first review to collate systematically all studies reporting on outcomes following MBU admission. While the evidence base is neither large nor methodologically robust, this review finds encouraging evidence that MBUs positively impact on maternal mental health, the mother-infant relationship, and possibly child development. However, due to the limitations of these studies, these conclusions are preliminary. The recommendations aim to support the growth of this emerging research literature to guide clinical practice.

\section{Acknowledgment}

We would like to thank Nadine Santos for her assistance as an independent rater.

\section{Disclosure}

The authors report no conflicts of interest in this work.

\section{References}

1. Bauer A, Parsonage M, Knapp M, Iemmi V, Adelaja B. The Costs of Perinatal Mental Health Problems. London: Centre for Mental Health and London School of Economics; 2014.

2. NHS England. NHS Standard Contract for Specialised Perinatal Mental Health Services. Leeds: NHS England; 2013.

3. Glangeaud-Freudenthal NM, Howard LM, Sutter-Dallay AL. Treatment - mother-infant inpatient units. Best Pract Res Clin Obstet Gynaecol. 2014;28(1):147-157.

4. Main TF. Mothers with children in a psychiatric hospital. Lancet. 1958;2(7051):845-847.

5. National Institute for Health and Care Excellence. Antenatal and Postnatal Mental Health: Clinical Management and Service Guidance. Manchester: NICE; 2014.

6. Scottish Intercollegiate Guidelines Network. Management of Perinatal Mood Disorders: A National Clinical Guideline. Edinburgh: SIGN; 2012.

7. Moher D, Liberati A, Tetzlaff J, Altman DG. Preferred reporting items for systematic reviews and meta-analyses: the PRISMA statement. Ann Intern Med. 2009;151(4):264-269.

8. Sirriyeh R, Lawton R, Gardner P, Armitage G. Reviewing studies with diverse designs: the development and evaluation of a new tool. J Eval Clin Pract. 2012;18(4):746-752.

9. Howard LM, Thornicroft G, Salmon M, Appleby L. Predictors of parenting outcome in women with psychotic disorders discharged from mother and baby units. Acta Psychiatr Scand. 2004;110(5):347-355.

10. Garfield P, Kent A, Paykel ES, Creighton FJ, Jacobson RR. Outcome of postpartum disorders: a 10 year follow-up of hospital admissions. Acta Psychiatr Scand. 2004;109(6):434-439.

11. Fisher J, Feekery C, Rowe H. Treatment of maternal mood disorder and infant behaviour disturbance in an Australian private mothercraft unit: a follow-up study. Arch Womens Ment Health. 2004;7(1):89-93. 
12. Reddy PD, Desai G, Hamza A, Karthik S, Ananthanpillai ST, Chandra PS. Enhancing mother infant interactions through video feedback enabled interventions in women with schizophrenia: a single subject research design study. Indian J Psychol Med. 2014;36(4):373-377.

13. Bilszta JL, Buist AE, Wang F, Zulkefli NR. Use of video feedback intervention in an inpatient perinatal psychiatric setting to improve maternal parenting. Arch Womens Ment Health. 2012;15(4):249-257.

14. Baker AA, Morison M, Game JA, Thorpe JG. Admitting schizophrenic mothers with their babies. Lancet. 1961;2(7196):237-239.

15. Beck AT, Steer RA, Brown GK. Beck Depression Inventory. Manual - Swedish version. Sandviken, Sweden: Psykologiförlaget; 2005.

16. Bardon D, Glaser YI, Prothero D, Weston DH. Mother and baby unit: psychiatric survey of 115 cases. Br Med J. 1968;2(5607):755-758.

17. Salmon MP, Abel K, Webb R, Warburton AL, Appleby L. A national audit of joint mother and baby admissions to UK psychiatric hospitals: an overview of findings. Arch Womens Ment Health. 2004;7(1): 65-70.

18. Salmon M, Abel K, Cordingley L, Friedman T, Appleby L. Clinical and parenting skills outcomes following joint mother-baby psychiatric admission. Aust N Z J Psychiatry. 2003;37(5):556-562.

19. Glangeaud-Freudenthal NM, Sutter AL, Thieulin AC, et al. Inpatient mother-and-child postpartum psychiatric care: factors associated with improvement in maternal mental health. Eur Psychiatry. 2011; 26(4):215-223.

20. Glangeaud-Freudenthal NM. Mother-baby psychiatric units (MBUs): national data collection in France and in Belgium (1999-2000). Arch Womens Ment Health. 2004;7(1):59-64.

21. Vliegen N, Casalin S, Luyten P, et al. Hospitalization-based treatment for postpartum depressed mothers and their babies: rationale, principles, and preliminary follow-up data. Psychiatry. 2013;76(2):150-168.

22. Spielberger CD, Gorsuch RL, Lushene RE. Manual for the State-Trait Anxiety Inventory. Palo Alto (CA): Consulting Psychologists; 1970.

23. Spielberger CD. State-Trait Anger Expression Inventory: Professional Manual. Odessa (FL): Psychological Assessment Resources; 1996.

24. Fontaine JRJ, Poortinga YH, Setiadi B, Markam SS. Cognitive structure of emotion terms in Indonesia and the Netherlands. Cogn Emot. 2002;16(1):61-86.

25. Vliegen N, Luyten P, Besser A, Casalin S, Kempke S, Tang E. Stability and change in levels of depression and personality: a follow-up study of postpartum depressed mothers that were hospitalized in a mother-infant unit. J Nerv Ment Dis. 2010;198(1):45-51.

26. Blatt SJ, D’Afflitti JP, Quinlan DM. Experiences of depression in normal young adults. J Abnorm Psychol. 1976;85(4):383-389.

27. Wan MW, Warburton AL, Appleby L, Abel KM. Mother and baby unit admissions: feasibility study examining child outcomes 4-6 years on. Aust N Z J Psychiatry. 2007;41(2):150-156.

28. Beck AT, Ward CH, Mendelson M, Mock J, Erbaugh JK. An inventory for measuring depression. Arch Gen Psychiatry. 1961;4(6) 561-571.

29. Hipwell AE, Goossens FA, Melhuish EC, Kumar R. Severe maternal psychopathology and infant-mother attachment. Dev Psychopathol. 2000; 12(2):157-175.

30. Meltzer-Brody S, Brandon AR, Pearson B, et al. Evaluating the clinical effectiveness of a specialized perinatal psychiatry inpatient unit. Arch Womens Ment Health. 2014;17(2):107-113.

31. Cox JL, Holden JM, Sagovsky R. Detection of postnatal depression. Development of the 10-item Edinburgh Postnatal Depression Scale. Br J Psychiatry. 1987;150(6):782-786.

32. Kroenke K, Spitzer RL, Williams JB. The PHQ-9. J Gen Intern Med. 2001;16(9):606-613

33. Spitzer RL, Kroenke K, Williams JB, Löwe B. A brief measure for assessing generalized anxiety disorder: the GAD-7. Arch Intern Med. 2006;166(10):1092-1097.

34. Mundt JC, Marks IM, Shear MK, Greist JM. The Work and Social Adjustment Scale: a simple measure of impairment in functioning. $\mathrm{Br}$ J Psychiatry. 2002;180(5):461-464.
35. Snellen M, Mack K, Trauer T. Schizophrenia, mental state, and motherinfant interaction: examining the relationship. Aust $N$ Z J Psychiatry. 1999;33(6):902-911.

36. Kay SR. Positive and Negative Syndromes in Schizophrenia: Assessment and Research. New York: Psychology; 1991.

37. Bergink V, Lambregtse-van Den Berg MP, Koorengevel KM, Kupka R, Kushner SA. First-onset psychosis occurring in the postpartum period: a prospective cohort study. J Clin Psychiatry. 2011;72(11):1531-1537.

38. Guy W. Early Clinical Drug Evaluation Unit Assessment Manual for Psychopharmacology. Revised. Washington: Department of Health, Education, and Welfare; 1976

39. Young RC, Biggs JT, Ziegler VE, Meyer DA. A rating scale for mania: reliability, validity and sensitivity. Br J Psychiatry. 1978;133(5): 429-435.

40. Noorlander Y, Bergink V, van den Berg MP. Perceived and observed mother-child interaction at time of hospitalization and release in postpartum depression and psychosis. Arch Womens Ment Health. 2008; 11(1):49-56.

41. Wittenborn JR. Psychiatric Rating Scales: Manual. New York: Psychological Corporation; 1955.

42. Kumar R, Hipwell AE. Development of a clinical rating scale to assess mother-infant interaction in a psychiatric mother and baby unit. $\mathrm{Br}$ J Psychiatry. 1996;169(1):18-26.

43. Brockington IF, Oates J, George S, et al. A screening questionnaire for mother-infant bonding disorders. Arch Womens Ment Health. 2001; 3(4):133-140

44. Hipwell AE, Kumar R. Maternal psychopathology and prediction of outcome based on mother-infant interaction ratings (BMIS). $\mathrm{Br}$ J Psychiatry. 1996;169(5):655-661.

45. Gibaud-Wallston J. Development and Utility of the Parenting Sense of Competence Scale. Nashville: John F Kennedy Center for Research on Education and Human Development; 1978.

46. Broussard ER, Hartner MS. Further considerations regarding maternal perception of the first born. In Hellmuth J, editor. Exceptional Infant: Studies in Abnormalities. Vol 2. New York: Brunner/Mazel; 1971: $432-449$.

47. Wan M, Green J. The impact of maternal psychopathology on child-mother attachment. Arch Womens Ment Health. 2009;12(3): 123-134.

48. Wolke D. Play Observation Scheme and Emotion Rating (POSER) Manual. Hatfield, UK: University of Hertfordshire; 1986.

49. Crittenden PM. CARE-Index Manual. Miami: Family Relations Institute; 2003.

50. Kenny M, Conroy S, Pariante CM, Seneviratne G, Pawlby S. Motherinfant interaction in mother and baby unit patients: before and after treatment. J Psychiatr Res. 2013;47(9):1192-1198.

51. Riordan D, Appleby L, Faragher B. Mother-infant interaction in postpartum women with schizophrenia and affective disorders. Psychol Med. 1999;29(4):991-995.

52. Murray L, Fiori-Cowley A, Hooper R, Cooper P. The impact of postnatal depression and associated adversity on early mother-infant interactions and later infant outcome. Child Dev. 1996;67(5):2512-2526.

53. Pawlby S, Fernyhough C, Meins E, Pariante C, Seneviratne G, Bentall R. Mind-mindedness and maternal responsiveness in infant-mother interactions in mothers with severe mental illness. Psychol Med. 2010; 40(11):1861-1869.

54. Meins E, Fernyhough C. Mind-Mindedness Coding Manual. Durham, UK: Durham University; 2006.

55. Fiese BH, Poehlmann J, Irwin M, Gordon M, Curry-Bleggi E. A pediatric screening instrument to detect problematic infant-parent interactions: initial reliability and validity in a sample of high-and low-risk infants. Infant Ment Health J. 2001;22(4):463-478.

56. Abel KM, Webb RT, Salmon MP, Wan MW, Appleby L. Prevalence and predictors of parenting outcomes in a cohort of mothers with schizophrenia admitted for joint mother and baby psychiatric care in England. J Clin Psychiatry. 2005;66(6):781-789; quiz 808-789. 
57. Milgrom J, Burrows GD, Snellen M, Stamboulakis W, Burrows K. Psychiatric illness in women: a review of the function of a specialist mother-baby unit. Aust N Z J Psychiatry. 1998;32(5):680-686.

58. Angermeyer MC, Dietrich S. Public beliefs about and attitudes towards people with mental illness: a review of population studies. Acta Psychiatr Scand. 2006;113(3):163-179.
59. Wan MW, Salmon MP, Riordan DM, Appleby L, Webb R, Abel KM. What predicts poor mother-infant interaction in schizophrenia? Psychol Med. 2007;37(4):537-546.

International Journal of Women's Health

Dovepress

\section{Publish your work in this journal}

The International Journal of Women's Health is an international, peerreviewed open-access journal publishing original research, reports, editorials, reviews and commentaries on all aspects of women's healthcare including gynecology, obstetrics, and breast cancer. The manuscript management system is completely online and includes

a very quick and fair peer-review system, which is all easy to use. Visit http://www.dovepress.com/testimonials.php to read real quotes from published authors.

Submit your manuscript here: http://www.dovepress.com/international-journal-of-womens-health-journal 